\title{
Current Directions in Research and Treatment of COVID-19
}

\author{
Karol Wróblewski ${ }^{1,2 *}$ \\ ${ }^{1}$ Department of Experimental and Clinical Pharmacology, University of Rzeszów, Poland; ${ }^{2}$ Laboratory for Innovative Research \\ in Pharmacology, University of Rzeszów, Kopisto 2a, 35-959 Rzeszów, Poland
}

\begin{abstract}
Severe acute respiratory syndrome coronavirus (SARS-CoV-2) is a novel coronavirus that emerged in Wuhan, China at the end of 2019. ${ }^{1}$ SARS-CoV-2 has spread fast all over the world due to its high transmissibility. SARS-CoV-2 can cause the disease known as coronavirus disease 2019 (COVID-19). Typical symptoms of COVID-19 include fever, fatigue, dry cough and dyspnea in severe cases. The elderly and people with co-morbidities are the demographic groups that are at higher risks to develop severe symptoms, such as acute respiratory distress syndrome, multiple organ dysfunction symdrome and others. However, many people with SARS-CoV-2 infection, mainly children and young adults, can be asymptomatic or mildly symptomatic. ${ }^{2}$
\end{abstract}

The COVID-19 pandemic is an international health problem. According to the World Health Organization, there have been over 76 milion confirmed cases of COVID-19 worldwide, including over 1.7 milion deaths as of Deccember 23, 2020. ${ }^{3}$ Knowledge of the virus and its infectivity, as well as the clinical characteristics of the disease and treatment methods have made rapid progress over the last year. ${ }^{2}$ However, a huge knowledge gap remains regarding the effects of this novel virus on the human body and the potential effective treatment strategies available for COVID-19.

To date, there are no proven effective therapies against COVID-19 or antivirals against SARS-CoV-2. One possible exception is remdesivir, which has been approved for treatment of COVID-19 by the Food and Drug Administration of the USA. However, some research findings give hope for the future. Currently, there are 459 therapeutic drugs in development for the treatment of COVID-19 and 372 in human clinical trials. Similarly, there are 187 vaccines in development and 62 in human trials. In particular, the SARS-CoV2 spiked the incidence of protein-based RNA vaccines and those produced by Pifzer and Moderna have been approved as an effective prevention in several countries. ${ }^{4}$ Potential therapeutic strategies against COVID-19 are shown in Figure 1.2 Remdesivir can significantly promote the recovery of some hospitalized COVID-19 patients, ${ }^{5}$ however, the therapeutic efficacy is unsatisfactory. ${ }^{6}$

A recent clinical trial by Simonovich et al. ${ }^{7}$ indicated that the use of convalescent plasma therapy in addition to standard treatment in patients with severe COVID-19 disease did not improve clinical outcomes or reduce mortality. This result may stem from

Abbreviations: SARS-CoV-2, severe acute respiratory syndrome coronavirus; COVID-19, coronavirus disease 2019.

Received: December 23, 2020; Revised: December 29, 2020; Accepted: January 08, 2021

*Correspondence to: Karol Wróblewski, Department of Experimental and Clinical Pharmacology, University of Rzeszów, Kopisto 2a, 35-959 Rzeszów, Poland. ORCID: http://orcid.org/0000-0002-8918-7056. Tel: + 48-178516889; E-mail: karolw222@ wp.pl

How to cite this article: Wróblewski K. Current Directions in Research and Treatment of COVID-19. J Explor Res Pharmacol 2020;000(000):000-000. doi: 10.14218/ JERP.2020.00041. some obvious limitations, such as the inclusion of one very severe COVID-19 patient. Therefore, additional trials should be performed for other clinical groups, including patients with mild to moderate COVID-19 to validate these results. Besides, such studies should evaluate the potential efficacy of passive immunotherapy applied before entry to the trial, and as soon as possible after diagnosis, mainly in patients with risk factors for severe disease.

The development of effective vaccines can be one of the most important approaches to prevent the virus from spreading and to reduce the incidence of COVID-19. The current clinical trials are necessary to validate the safety and effectiveness of different vaccines in a large and diverse population. ${ }^{8}$ Recent results indicate that two mRNA vaccines conferred $95 \%$ and $94.1 \%$ protection against COVID-19 in adults 9,10 and have been approved for human vaccination in several countries. However, due to the limitations of restrictive vaccine transportation and limited production, as well as their high cost, these vaccines may be insufficient to prevent the continued spread of COVID-19 across the world. Hence, safer and cheaper vaccines with convenient transportation methods are urgently needed.

During the past year, there have been significant advances in the diagnosis of SARS-CoV2 infection and clinical COVID-19. Various therapeutic agents have also been tested that can fight against the COVID-19. Nevertheless, there is still a huge gap in knowledge regarding the biology of SARS-CoV2 and the pathogenesis of COVID-19, as well as the pharmacological mechanisms underlying the effects of various therapeutic drugs. The effectiveness of existing therapies for treating COVID-19 is very limited. Accordingly, there is an urgent requirement for the development of effective drugs and vaccines to eradicate the COVID-19 pandemic.

\section{Acknowledgments}

None.

Funding

None.

Conflict of interest

The author declares that there is no conflict of interest.

\section{References}

[1] Wu JT, Leung K, Leung GM. Nowcasting and forecasting the potential 


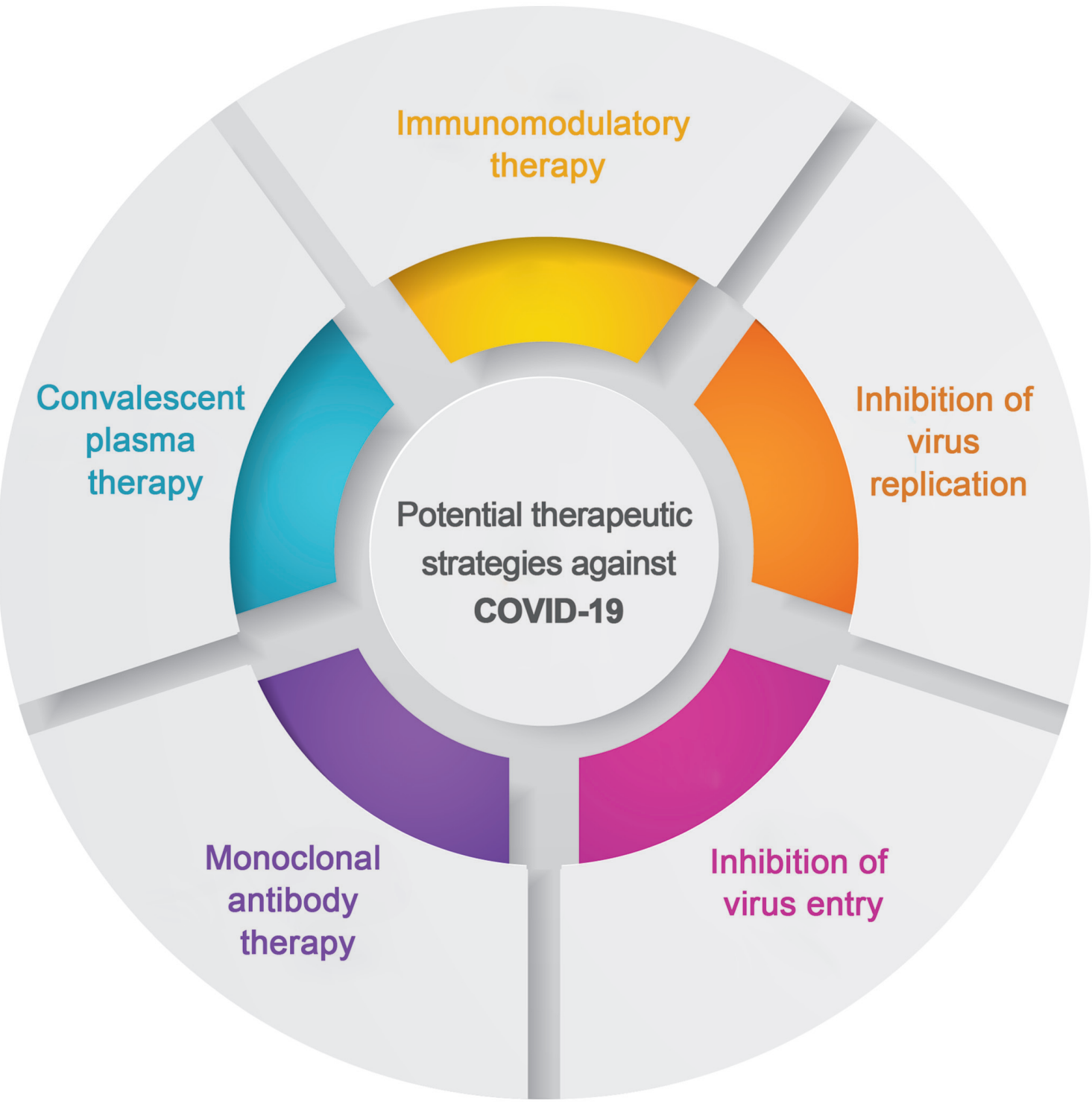

Fig. 1. Potential therapeutic strategies against COVID-19.

domestic and international spread of the 2019-nCoV outbreak originating in Wuhan, China: a modelling study. Lancet 2020;395(10225): 689-697. doi:10.1016/S0140-6736(20)30260-9.

[2] Hu B, Guo H, Zhou P, Shi ZL. Characteristics of SARS-CoV-2 and COVID-19. Nat Rev Microbiol 2020:1-14. doi:10.1038/s41579-02000459-7.

[3] WHO. WHO Coronavirus Disease (COVID-19) Dashboard. Available from: https://covid19.who.int. Accessed December 23, 2020.

[4] bioRENDER. COVID-19 vaccine and therapeutics tracker. Available from: https://biorender.com/covid-vaccine-tracker. Accessed December 23, 2020.

[5] Beigel JH, Tomashek KM, Dodd LE, Mehta AK, Zingman BS, Kalil AC, et al. Remdesivir for the Treatment of Covid-19 - Final Report. N Engl J Med 2020;383(19):1813-1826. doi:10.1056/NEJMoa2007764.

[6] Enoki $Y$, Igarashi $Y, W a t a b e ~ Y, ~ H o n m a ~ K$, Suzuki $Y$, Hayashi $Y$, et al. Remdesivir for the treatment of coronavirus COVID-19: A meta-analysis of randomised controlled trials. J Glob Antimicrob Resist 2020;24: 81-82. doi:10.1016/j.jgar.2020.11.022.

[7] Simonovich VA, Burgos Pratx LD, Scibona P, Beruto MV, Vallone MG, Vázquez $\mathrm{C}$, et al. A Randomized Trial of Convalescent Plasma in Covid-19 Severe Pneumonia. N Engl J Med 2020. doi:10.1056/NEJMoa2031304.

[8] Rawat K, Kumari P, Saha L. COVID-19 vaccine: A recent update in pipeline vaccines, their design and development strategies. Eur Pharmacol 2020;892:173751. doi:10.1016/j.ejphar.2020.173751.

[9] Polack FP, Thomas SJ, Kitchin N, Absalon J, Gurtman A, Lockhart $\mathrm{S}$, et al. Safety and Efficacy of the BNT162b2 mRNA Covid-19 Vaccine. N Engl J Med 2020;383(27):2603-2615. doi:10.1056/NEJMoa 2034577.

[10] Mahase E. Covid-19: Moderna applies for US and EU approval as vaccine trial reports 94.1\% efficacy. BMJ 2020;371:m4709. doi:10.1136/ bmj.m4709. 